\title{
Treatment of Osteoporotic Vertebral Compression Fracture by Precise Injection and Staged Perfusion of Bone Cement Kyphoplasty: A Comparison Study
}

Jian Huang ( $\sim 67866987 @ q q . c o m$ )

Haikou Hospital of Traditional Chinese Medicine https://orcid.org/0000-0002-5434-1148

Jun Huang

The 928th Hospital of the Chinese People's Liberation Army

Ming Chen

Haikou Hospital of Traditional Chinese Medicine

Zongbo Zhou

Haikou Hospital of Traditional Chinese Medicine

Zhifu Lu

Haikou Hospital of Traditional Chinese Medicine

Chuangong Fu

Haikou Hospital of Traditional Chinese Medicine

Research article

Keywords: bone cement, precise injection, staged perfusion, kyphoplasty, osteoporotic vertebral compression fracture

Posted Date: August 30th, 2021

DOl: https://doi.org/10.21203/rs.3.rs-809547/v1

License: (c) (i) This work is licensed under a Creative Commons Attribution 4.0 International License.

Read Full License 


\section{Abstract}

Study design: Retrospective cohort study.

Objective: To explore the clinical effect of precise injection and staged perfusion of bone cement kyphoplasty in the treatment of osteoporotic vertebral compression fracture.

Methods: 110 patients treated with kyphoplasty from January 2020 to June 2021 were selected and divided into experimental group $(n=55)$ and control group $(n=55)$ according to different surgical methods. The experimental group was treated with precise injection and staged perfusion of bone cement kyphoplasty, while the control group was treated with traditional kyphoplasty. The operation time,intraoperative blood loss and amount of bone cement injection were recorded. The pain improvement was evaluated by VAS score. The operation effect were evaluated by anterior height of injured vertebral body, middle height of injured vertebral body, wedge angle of injured vertebral body and distribution grade of bone cement. The incidence of surgical complications was evaluated by the number of bone cement leakage.

Results: There were no significant difference in the operation time, intraoperative blood loss and the amount of bone cement injection in two groups. There were no significant difference in VAS scores at $2 \mathrm{~h}$, $4 \mathrm{~h}$ and $48 \mathrm{~h}$ after operation between the two groups $(P>0.05)$; There were no significant difference in the ratio of anterior height of injured vertebral body between the two groups on the third day after operation and the last follow-up $(P>0.05)$; There were no significant difference in the ratio of middle height of injured vertebral body between the two groups on the third day after operation and the last follow-up $(P>$ $0.05)$; There were no significant difference in wedge angle of injured vertebral body between the two groups at the third day after operation and the last follow-up $(P>0.05)$. There was significant difference in the distribution grade of bone cement between the two groups $(P<0.01)$; There was significant difference in the number of bone cement leakage between the two groups $(P<0.01)$, In cases of bone cement leakage, there were 1 case of type $C, 1$ case of type $S$ in the experimental group, 7 cases of type $C$ and 2 cases of type $S$ in the control group.

Conclusion: Precise injection and staged perfusion of bone cement kyphoplasty in the treatment of osteoporotic vertebral compression fracture can effectively reduce surgical complications and improve surgical efficacy.

\section{Backgroud}

OVCF is a pathological fracture. It is a vertebral compression fracture based on osteoporosis.

Osteoporosis is a systemic metabolic bone disease with decreased bone mass per unit volume and bone strength due to the imbalance between bone tissue formation and absorption. It is common in postmenopausal women and can also be secondary to inflammation and endocrine diseases [1, 2]. Bone loss and osteopenia are a continuous process. They are asymptomatic in the early stage. Osteoporosis is found only when fractures occur and patients see a doctor [3]. Vertebral fracture is the most common 
fracture in patients with osteoporosis. Patients often complain about low back pain and dare not move [4], which is often caused by minor trauma and it belongs to low-energy fracture. At present, vertebroplasty or kyphoplasty is the first choice in treatment of OVCF. Through the percutaneous establishment of working channel, bone cement is injected into the vertebral body from the working channel, and the anchoring effect of bone cement in the vertebral body, or the exothermic polymerization and toxic effects of bone cement destroy nerve endings and inflammatory pain causing factors to achieve analgesic effect. But there are complications of bone cement leakage. Under the premise of existing equipment, how to inject sufficient bone cement to ensure clinical efficacy without leakage is a topic of concern for clinicians. In this study, precise injection and Staged perfusion of bone cement Kyphoplasty were used to treat OVCF, in order to find a method that can not only achieve clinical efficacy, but also reduce the risk of bone cement leakage.

\section{Materials And Methods}

\section{Patient selection method}

Inclusion criteria include the following: (a) back and waist pain, limited activity; (b) MRI displayed fresh

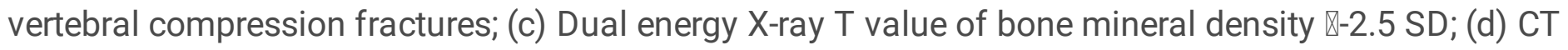
scan diagnosed as thoracolumbar flexion compression fractures, Denis type A [6]; (e) ASIA Grade E; (f) 60 years and older; $(\mathrm{g})$ the responsible vertebral body of patient was a single segment.

Exclusion criteria include the following: (a) patients with other fractures; (b) patients with bone cement allergy; (c) patients with nerve injury and progressive aggravation; (d) patients with osteomyelitis and epidural cyst; (e) Patients with vertebral bone metastasis; (f) patients with coagulation dysfunction.

\section{General information}

110 patients hospitalized for kyphoplasty from January 2020 to June 2021 were selected. According to different treatment methods, they were divided into 55 cases in the experimental group and 55 cases in the control group.

\section{Surgical technique}

All operations were performed by the chief surgeon of spine surgery. All patients were treated with local infiltration anesthesia ${ }^{[7]}$. All patients were in prone position, with pillows on the chest and ilium ${ }^{[8]}$. The pedicle of the responsible vertebral body was located and marked by C-arm fluoroscopy. The 10'a clock and 2'a clock positions of the pedicle shadow on both sides of the responsible vertebral body were used as puncture points. The puncture points by $\mathrm{C}$-arm fluoroscopy was good, Maintain the appropriate lateral tilting angle and upper tilting inclination angle, continue to knock the needle inward. C-arm fluoroscopy showed that the needle tip had reached the medial edge of pedicle shadow in the anterior and posterior 
position, and the needle tip had reached the posterior edge of vertebral body in the lateral position. Continue to knock the needle inward for $3 \mathrm{~mm}$, removed the inner core of the needle, and had established the working channel. A bone drill was built into the working channels through both sides to expand the bone channel in the vertebral body, and then a balloon was placed to expand. The edge of the balloon was close to the upper and lower endplates or reached the cortex around the vertebral body, or the expansion stoped when the vertebral fracture has been reset.

In the control group,inserted a bone cement filling tube with a forward opening through the working channel, and injected an appropriate amount of bone cement when the bone cement enters the "wire drawing stage". After filling, took out the filling tube and working channel. Sutured the incision.

In the experimental group, if it belonged to Denis type $A \otimes$ fracture, selected the bone cement filling tube with forward opening. If it belonged to Denis type $A \otimes-\otimes$ fracture, selected the bone cement filling tube with opening to the side. Placed the bone cement filling tube into the collapse part of the bone cortex, injected the bone cement when the bone cement was in the "dough stage". The C-arm fluoroscopy showed that the bone cortex breach of the vertebral body has been closed, and the bone cement was mixed again, injected the bone cement into all directions of the vertebral body when the bone cement was in the "wire drawing stage". The $\mathrm{C}$-arm fluoroscopy showed that the bone cement dispersion was satisfactory. After filling, took out the filling tube and working channel. Sutured the incision. (Fig. 1, 2).

\section{Postoperative managements}

The two groups were moved under the protection of thoracolumbar brace on the second day after operation. Change dressing for incision on time.

\section{Efficacy evaluation}

All patients were followed up for at leat 6 months after treatment. The operation time, intraoperative blood loss and the amount of bone cement injection of all patients were recorded. Intraoperative blood loss $=$ (preoperative hemoglobin - postoperative hemoglobin) $/$ preoperative hemoglobin $\times 100 \%$. VAS pain score standard ${ }^{[9]}$ was used to evaluate the improvement of pain. From 0 to 10 points, the higher the score, the more obvious the pain. VAS scores before operation, $2 \mathrm{~h}, 4 \mathrm{~h}$ and $48 \mathrm{~h}$ after operation were recorded. The vertical height of the anterior edge of the upper and lower endplates in the median sagittal plane of the vertebral body was measured by lateral X-ray film ${ }^{[10]}$. The ratio of anterior height of injured vertebral body $=$ (anterior height of injured vertebral body / average height of anterior edge of upper and lower vertebral body of injured vertebral body) $\times 100 \%$. The anterior height of injured vertebral body was recorded before operation, 3 days after operation and the last follow-up. The vertical height of the middle of the upper and lower endplates in the median sagittal plane of the vertebral body was measured by lateral X-ray film. The ratio of middle height of injured vertebral body = (middle height of injured vertebral body / average height of middle of upper and lower vertebral body of injured vertebral body) $\times 100 \%$. The 
middle height of injured vertebral body was recorded before operation, 3 days after operation and the last follow-up. The angle between the extension lines of the upper and lower endplates in the median sagittal plane of the vertebral body was measured by lateral X-ray film. The wedge angle of injured vertebral body was recorded before operation, 3 days after operation and the last follow-up [11]. The distribution of bone cement was evaluated by distribution grade. The distribution grade of bone cement was divided into four grades: Grade I, bone cement was filled tightly, and the area of bone cement on anteroposterior position and / or lateral position X-ray films accounts for $50 \% \sim 75 \%$ of the area of vertebral body; grade II, bone cement was spongy filling, and the area of bone cement on anteroposterior position and / or lateral position X-ray films accounts for $50 \% \sim 75 \%$ of the area of vertebral body; grade III, bone cement filling was dense, and the area of bone cement on anteroposterior position and lateral position X-ray films was greater than $75 \%$ of the area of vertebral body; grade IV, bone cement was spongy filling, and the area of bone cement on anteroposterior position and lateral position X-ray films was greater than $75 \%$ of the area of vertebral body. The distribution grade of bone cement in the two groups was evaluated after operation. The leakage of bone cement was classified according to the method proposed by Yeom et al. [12]: Type B, the bone cement leaked along the paravertebral vein to the posterior edge of the vertebral body and was relatively symmetrically distributed at the posterior edge of the vertebral body; type $C$, bone cement leaked along the cortical defect, and can leak around the vertebral body, intervertebral disc and spinal canal; type $S$, bone cement leaked around the vertebral body through segmental vein. As long as the bone cement exceeded the edge of bone cortex, it was bone cement leakage. The number and classification of bone cement leakage in the two groups were recorded after operation.

\section{Statistical methods}

SPSS 26.0 was used for data analysis. The measurement data were expressed by mean \pm standard deviation. For intergroup comparison, variance homogeneity $F$ test was used first, then independent sample $t / t^{\prime}$ test was used, and paired sample $t$ test was used for intragroup comparison. The count data were expressed by the number of cases and percentage, and the comparison of counting data were performed by chi-square test, Mann Whitney $U$ rank sum test was used for comparison of grade data. Test level $a=0.05$, bilateral test.

\section{Results}

\section{General results}

There were no significant difference in gender, age,clinical manifestations and responsible vertebral body between the two groups (Table 1). 
Table 1

Patient characteristics

\begin{tabular}{|llll|}
\hline & experimental group & control group & $P \nabla$ \\
\hline Number & 55 & 55 & \\
\hline Gender (male to female) & $12: 43$ & $14: 41$ & 0.483 \\
\hline Age $(y r \pm S D)$ & $69.47 \pm 6.90$ & $70.38 \pm 7.25$ & 0.374 \\
\hline Clinical manifestations & & & \\
\hline Pain & 55 & 55 & 0.364 \\
\hline Percussion pain & 55 & 55 & 0.312 \\
\hline Constipation & 37 & 39 & 0.397 \\
\hline Responsible vertebral body & & 30 & 0.532 \\
\hline $\begin{array}{l}\text { Thoracic vertebra } \\
\text { Lumbar vertebra }\end{array}$ & 32 & 25 & 0.498 \\
\hline $\begin{array}{l}\nabla P \text { value of the age is calculated by variance analysis;others are calculated by chi-square test } \\
y r \text { hour, } S D \text { standard deviation }\end{array}$ & 23 & \\
\hline
\end{tabular}

Comparison of operation time, intraoperative blood loss and amount of bone cement injection There were no significant difference in operation time, intraoperative blood loss and amount of bone cement injection between the two groups (Table 2).

Table 2

Comparison of operation time,intraoperative blood loss and amount of bone cement injection in two groups

\begin{tabular}{|lllll|}
\hline & experimental group & control group & $\boldsymbol{t} / \boldsymbol{t}^{\prime}$ & $\boldsymbol{P}$ \\
\hline operation time(min) & $32 \pm 4.46$ & $29 \pm 5.17$ & 0.782 & 0.528 \\
\hline intraoperative blood loss(\%) & $1.91 \pm 0.84$ & $1.93 \pm 0.69$ & 0.693 & 0.586 \\
\hline amount of bone cement injection $(\mathrm{ml})$ & $5.5 \pm 1.21$ & $5.2 \pm 1.16$ & 0.572 & 0.607 \\
\hline Values are mean \pm SD & & & & \\
\hline
\end{tabular}

\section{Comparison of VAS scores}

There was no significant difference in VAS score between the two groups on preoperation. In each group,there were significant difference in VAS scores between the preoperation and $2 \mathrm{~h}, 4 \mathrm{~h}$ and $48 \mathrm{~h}$ after 
operation. But there were no significant difference in VAS scores between the two groups at $2 \mathrm{~h}, 4 \mathrm{~h}$ and 48h after operation (Table 3 ).

Table 3

Comparison of VAS scores in two groups

\begin{tabular}{|lllll|}
\hline & experimental group & control group & $t / \boldsymbol{t}^{\prime}$ & $\boldsymbol{P}$ \\
\hline Preoperation & $8.34 \pm 0.27$ & $8.24 \pm 0.67$ & 0.296 & 1.108 \\
\hline 2h after operation & $3.41 \pm 0.34^{*}$ & $3.43 \pm 0.41^{*}$ & 0.381 & 0.864 \\
& $t=4.075, P<0.05$ & $t=4.573, P<0.05$ & & \\
\hline 4h after operation & $1.16 \pm 0.59^{*}$ & $1.26 \pm 0.82^{*}$ & 0.423 & 0.706 \\
& $t=5.423, P<0.01$ & $t=5.590, P<0.01$ & & \\
\hline 48h after operation & $0.15 \pm 0.09^{*}$ & $0.17 \pm 0.07^{*}$ & 0.392 & 0.792 \\
& $t=5.897, P<0.01$ & $t=5.697, P<0.01$ & & \\
\hline Values are mean \pm SD & & & & \\
\hline *Statistically significant & & & \\
\hline
\end{tabular}

\section{Comparison of ratio of anterior height of injured vertebral body}

There was no significant difference in the ratio of anterior height of injured vertebral body between the two groups before operation. In each group, there were significant difference in the ratio of anterior height of injured vertebral body between the preoperation and 3 days after operation, last follow-up. But there were no significant difference in the ratio of anterior height of injured vertebral body between the two groups at 3 days after operation and last follow-up (Table 4). 
Table 4

Comparison of ratio of anterior height of injured vertebral body in two groups

\begin{tabular}{|lllll|}
\hline & experimental group & control group & $t / \boldsymbol{t}^{\prime}$ & $P$ \\
\hline Preoperation & $47.36 \pm 6.16$ & $46.38 \pm 5.37$ & 0.793 & 0.526 \\
\hline 3 days after operation & $85.32 \pm 6.17^{*}$ & $84.97 \pm 5.74^{\star}$ & 0.375 & 0.964 \\
& $t=4.157, P<0.05$ & $t=4.273, P<0.05$ & & \\
\hline Last follow-up & $86.43 \pm 6.57^{\star}$ & $87.67 \pm 6.07^{\star}$ & 0.526 & 0.638 \\
& $t=4.752, P<0.05$ & $t=4.586, P<0.05$ & & \\
\hline Values are mean \pm SD & & & & \\
\hline *Statistically significant & & & & \\
\hline
\end{tabular}

\section{Comparison of ratio of middle height of injured vertebral body}

There was no significant difference in the ratio of middle height of injured vertebral body between the two groups before operation. In each group, there were significant difference in the ratio of middle height of injured vertebral body between the preoperation and 3 days after operation, last follow-up. But there were no significant difference in the ratio of middle height of injured vertebral body between the two groups at 3 days after operation and last follow-up (Table 5).

Table 5

Comparison of ratio of middle height of injured vertebral body in two groups

\begin{tabular}{|lllll|}
\hline & experimental group & control group & $t / \boldsymbol{t}^{\prime}$ & $P$ \\
\hline Preoperation & $53.56 \pm 6.84$ & $52.52 \pm 5.62$ & 0.783 & 0.528 \\
\hline 3 days after operation & $91.74 \pm 3.37^{\star}$ & $89.54 \pm 3.44^{\star}$ & 0.369 & 0.994 \\
& $t=4.590, P<0.05$ & $t=4.563, P<0.05$ & & \\
\hline Last follow-up & $89.63 \pm 4.52^{\star}$ & $87.75 \pm 5.64^{\star}$ & 0.471 & 0.674 \\
& $t=5.011, P<0.01$ & $t=5.358, P<0.01$ & & \\
\hline Values are mean \pm SD & & & & \\
\hline *Statistically significant & & & & \\
\hline
\end{tabular}

\section{Comparison of wedge angle of injured vertebral body}


There was no significant difference in the wedge angle of injured vertebral body between the two groups before operation. In each group, there were significant difference in the wedge angle of injured vertebral body between the preoperation and 3 days after operation, last follow-up. But there were no significant difference in the wedge angle of injured vertebral body between the two groups at 3 days after operation and last follow-up (Table 6).

Table 6

Comparison of wedge angle of injured vertebral body in two groups

\begin{tabular}{|lllll|}
\hline & experimental group & control group & $t / \boldsymbol{t}^{\prime}$ & $\boldsymbol{P}$ \\
\hline Preoperation & $24.41 \pm 2.67$ & $25.53 \pm 2.35$ & 0.337 & 1.042 \\
\hline 3 days after operation & $3.46 \pm 1.13^{\star}$ & $3.73 \pm 1.05^{\star}$ & 0.246 & 1.176 \\
& $t=4.731, P<0.05$ & $t=4.891, P<0.05$ & & \\
\hline Last follow-up & $4.22 \pm 1.07^{*}$ & $4.13 \pm 1.09^{\star}$ & 0.389 & 0.827 \\
& $t=4.273, P<0.05$ & $t=4.667, P<0.05$ & & \\
\hline Values are mean \pm SD & & & & \\
\hline *Statistically significant & & & & \\
\hline
\end{tabular}

\section{Comparison of the distribution grade of bone cement and the number of bone cement leakage}

There were significant difference in the distribution grade of bone cement and the number of bone cement leakage between the two groups. In cases of bone cement leakage, there were 1 case of type $C, 1$ case of type $S$ in the experimental group, 7 cases of type $C$ and 2 cases of type $S$ in the control group. 
Table 7

Comparison of the distribution grade of bone cement and the number of bone cement leakage in two groups

\begin{tabular}{|c|c|c|c|c|}
\hline & experimental group & control group & & $P \nabla$ \\
\hline Distribution grade of bone cement & & & $U=392.00$ & $0.004 *$ \\
\hline Grade I & $4(7.3)$ & $11(20.0)$ & & \\
\hline Grade II & $7(12.7)$ & 18(32.7) & & \\
\hline Grade III & $10(18.2)$ & $14(25.5)$ & & \\
\hline Grade IV & $34(61.8)$ & $12(21.8)$ & & \\
\hline Number of bone cement leakage & & & - & $0.007 *$ \\
\hline Leakage & 2 & 9 & & \\
\hline No leakage & 53 & 46 & & \\
\hline Leakage rate(\%) & 3.6 & 16.4 & & \\
\hline \multicolumn{5}{|c|}{$\begin{array}{l}\nabla P \text { value of the distribution grade of bone cement is calculated by Mann Whitney } \mathrm{U} \text { rank sum } \\
\text { test; another is calculated by chi-square test }\end{array}$} \\
\hline
\end{tabular}

\section{Discussion}

OVCF is a common type of fracture in the middle-aged and elderly. In China, osteoporotic patients account for $15 \%$ of the population over the age of 50 , of which 1 / 3 are accompanied by vertebral fracture, and the risk of vertebral fracture increases with age [13, 14]. OVCF is a spinal fracture induced by systemicabnormal bone metabolism, decreased bone mass and increased bone fragility [15].

Pathologically, the vertebral body collapses and the height decreases, which affects the stability of the spine and is prone to kyphosis, resulting in the patient's shortening and hunchback deformity, affecting daily life [16]. At this stage, vertebroplasty and kyphoplasty are mostly used in the treatment of OVCF [17]. Both of them locate the body surface projection of pedicle through C-arm fluoroscopy in preoperative, puncture with puncture needle, establish working channel, and inject bone cement through working channel [18]. However, after the establishment of the working channel in the kyphoplasty, drilled the vertebral body with a bone drill, expanded with a balloon, and finally injected with bone cement. Because kyphoplasty forms a cavity in the vertebral body through balloon expansion, and the cancellous bone around the cavity is compacted, which can reduce the risk of bone cement leakage to a certain extent, and effectively restore the vertebral body height and avoid kyphosis deformity through balloon expansion, kyphoplasty is more widely used in clinic. For primary OVCF, kyphoplasty can be selected for treatment if pain can not be relieved or related complications caused by long-term bed rest can not be 
prevented [19]; kyphoplasty can also be selected to improve the pain symptoms of malignant tumor vertebral bone metastasis or benign tumor $[20,21]$.

In this study, after puncturing and the working channel established in the experimental group, the bone cement filling tube was inserted according to the specific position of the fracture collapse. If the vertebral forearm fracture collapsed, the bone cement filling tube with forward opening was selected. If the upper and lower final plates of the vertebral body collapsed, the bone cement filling tube with side opening was selected and the bone cement was injected accurately in stages. Firstly, the cortical breach was effectively closed by injecting bone cement when it was in " dough stage ", and then the fractured vertebral body was effectively filled by injecting bone cement when it was in "wire drawing stage ". It can not only reduce the risk of bone cement leakage, but also form the effective diffusion of bone cement in the vertebral body. Through comparison, it was found that the distribution grade of bone cement and the number of bone cement leakage in the experimental group with precisen injection and staged perfusion of bone cement were better than those in the control group without precise injection and staged perfusion of bone cement, while there were no significant difference in the operation time, intraoperative blood loss,amount of bone cement injection, VAS pain score, ratio of anterior and middle height of injured vertebral body and wedge angle of injured vertebral body between the two groups. Therefore, precise injection and staged perfusion of bone cement kyphoplasty in the treatment of OVCF can effectively reduce surgical complications and improve surgical efficacy.

However, the sample size of this study was small and lack of relevant laboratory data. In the future work, we will continue to expand the sample size, include more research objects, do a good job in long-term follow-up, and carry out biomechanical research under laboratory conditions, return clinical research to fundamental research, so as to serve the clinic.

\section{Conclusion}

Precise injection and staged perfusion of bone cement kyphoplasty in the treatment of OVCF can effectively reduce surgical complications and improve surgical efficacy, so it can be widely used in clinic.

\section{Abbreviations}

OVCF: osteoporotic vertebral compression fractures; MRI: Magnetic resonance imaging; CT: Computer tomography; ASIA: American spinal injury association; VAS: Visual analog scale.

\section{Declarations}

\section{Acknowledgements}

Not applicable. 


\section{Authors' contributions}

The author(s) read and approved the final manuscript.

\section{Funding}

Supported by Hainan Provincial Natural Science Foundation of China(820RC787).

\section{Availability of data and materials}

Not applicable.

\section{Ethics approval and consent to participate}

This study was approved by the ethics committee of the Haikou Hospital of Traditional Chinese Medicine. Informed consent was obtained from all individual participants included in the study.

\section{Consent for publication}

Not applicable.

\section{Competing interests}

The authors declare that they have no competing interests.

\section{Author details}

1Department of Orthopedics, Haikou Hospital of Traditional Chinese Medicine, 2 Poxiang Road, Longhua District, Haikou City, Hainan Province, People's Republic of China. ${ }^{2}$ Department of Anesthesiology, The 928th Hospital of the Chinese People's Liberation Army, 100 Longkun South Road, Qiongshan District, Haikou City, Hainan Province, People's Republic of China.

\section{References}

1. Que MQ, Shi JS, Huang YJ, et al. Mesh meta analysis of different drugs in the treatment of primary osteoporosis [J]. China tissue engineering research. 2020;24(35):5715-22.

2. Locantore P, Del Gatto V, Gelli S, et al. The Interplay between Immune System and Microbiota in Osteoporosis[J]. Mediators Inflamm 2020 Feb. 2020;26:3686749. doi:10.1155/2020/3686749. 
3. Zhong YM, He BK, Wu ZT, et al. Jack vertebral expander kyphoplasty in the treatment of osteoporotic vertebral compression fractures: an effective and safe meta-analysis [J]. China tissue engineering research. 2022;26(03):510-6.

4. Rao HJ, Wang DG, Huang LB, et al. PKP and zoledronic acid in the treatment of postmenopausal osteoporotic vertebral fractures [J]. Chinese Journal of Orthopaedic Surgery. 2021;29(12):1136-8.

5. Li Q, Long X, Wang Y, et al. Clinical observation of two bone cement distribution modes after percutaneous vertebroplasty for osteoporotic vertebral compression fractures.[J]. BMC Musculoskelet Disord. 2021;22(1):577-7.

6. Panjabi MM, Oxland TR, Kifune $M$, et al. Validity of the three column theory of thoracolumbar fractures. A biomechanic investigation. Spine. 1995;20:1122-7.

7. Liu LH, Wang JG, Wang XJ, et al. Lateral pedicle infiltration anesthesia for unilateral puncture vertebroplasty [J]. Journal of local interpretation surgery. 2017;26(03):178-80.

8. Huang J, Zhou LM, Yan ZD, et al. Effect of manual reduction and indirect decompression on thoracolumbar burst fracture: a comparison study.[J]. J Orthop Surg Res. 2020;15(1):532-2.

9. Agarwal N, Choi P, Sekula R. Minimally Invasive Spine Surgery for Unstable Thoracolumbar Burst Fractures: A Case Series[J]. Surgery Journal. 2016;02(04):e131-8.

10. Liu H, Zhao HR, Deng WX, et al. Experience of transpedicular and traumatic vertebral internal fixation in the treatment of thoracolumbar vertebral fractures [J]. Journal of practical orthopedics. 2011;17(11):1010-2.

11. Zhu Y, Chen LW, Wang SL, et al. Imaging analysis of manual reduction combined with PVP in the treatment of severe osteoporotic vertebral compression fractures [J]. Chinese Journal of bone joint injury. 2021;36(03):256-8.

12. Yeom JS, Kim WJ, Choy WS,et al. Leakage of cement in percutaneous transpedicular vertebroplasty for painful osteoporotic compression fractures[J]. J Bone Joint Surg Br. 2003;85(1):83-9. DOI:10.1302/0301-620x.85b1.13026.

13. Bu B, bu LL, Wang Y. Effects of zoledronic acid combined with Bushen Huoxue Decoction on bone mineral density and biochemical indexes of bone metabolism in patients with osteoporotic vertebral compression fracture during postoperative recovery [J]. Chinese Journal of surgery of integrated traditional Western medicine. 2021;27(02):233-6.

14. Wang J, Yang BH, Li HP. Risk factors analysis of osteoporotic lumbar vertebral compression fracture [J]. Chinese Journal of orthopedics traumatology. 2021;29(08):29-33.

15. Zhao L, Yang XM, Zhang Y. The Clinical Effect of Zoledronic Acid Combined with Teriparatide in Perverting Recurrent Fracture of Osteoporotic Vertebral Compressive Fractures in the Elderly after Percutaneous Kyphoplasty [J]. Surgical Science. 2021;12(06):161-73.

16. Tang GD, Yang QQ, Hou JT, et al. Comparison of unilateral and bilateral kyphoplasty for osteoporotic vertebral fractures [J]. Chinese Journal of Orthopaedic Surgery. 2021;29(10):939-42.

17. Zhang Z, Lin XS, Han LJ, et al. Effect of Fuyuan cream Acupoint Application on residual low back pain after PKP in patients with osteoporotic vertebral compression fracture [J]. Chinese Journal of 
osteoporosis. 2020;26(10):1509-13.

18. Li Q, Li J, Luan J, et al. Distribution of bone cement during angle vertebroplasty in the treatment of thoracolumbar osteoporotic compression fractures [J]. China tissue engineering research. 2021;25(16):2466-71.

19. Zhang ZL, Jing QM, Qiao R, et al. Risk factors of new fractures of adjacent vertebral bodies after percutaneous vertebroplasty for osteoporotic vertebral compression fractures [J]. Chinese Journal of repair reconstruction surgery. 2021;35(01):20-5.

20. Zhang QC, Zhang XS. Research status of minimally invasive treatment of spinal metastatic tumors [J]. Chinese Journal of Orthopaedic Surgery. 2021;29(03):245-8.

21. Zhao XS, Chu XD, Liu XW. Observation on the efficacy of percutaneous kyphoplasty in the treatment of spinal metastases [J]. China cancer clinic rehabilitation. 2019;26(06):690-3.

\section{Figures}

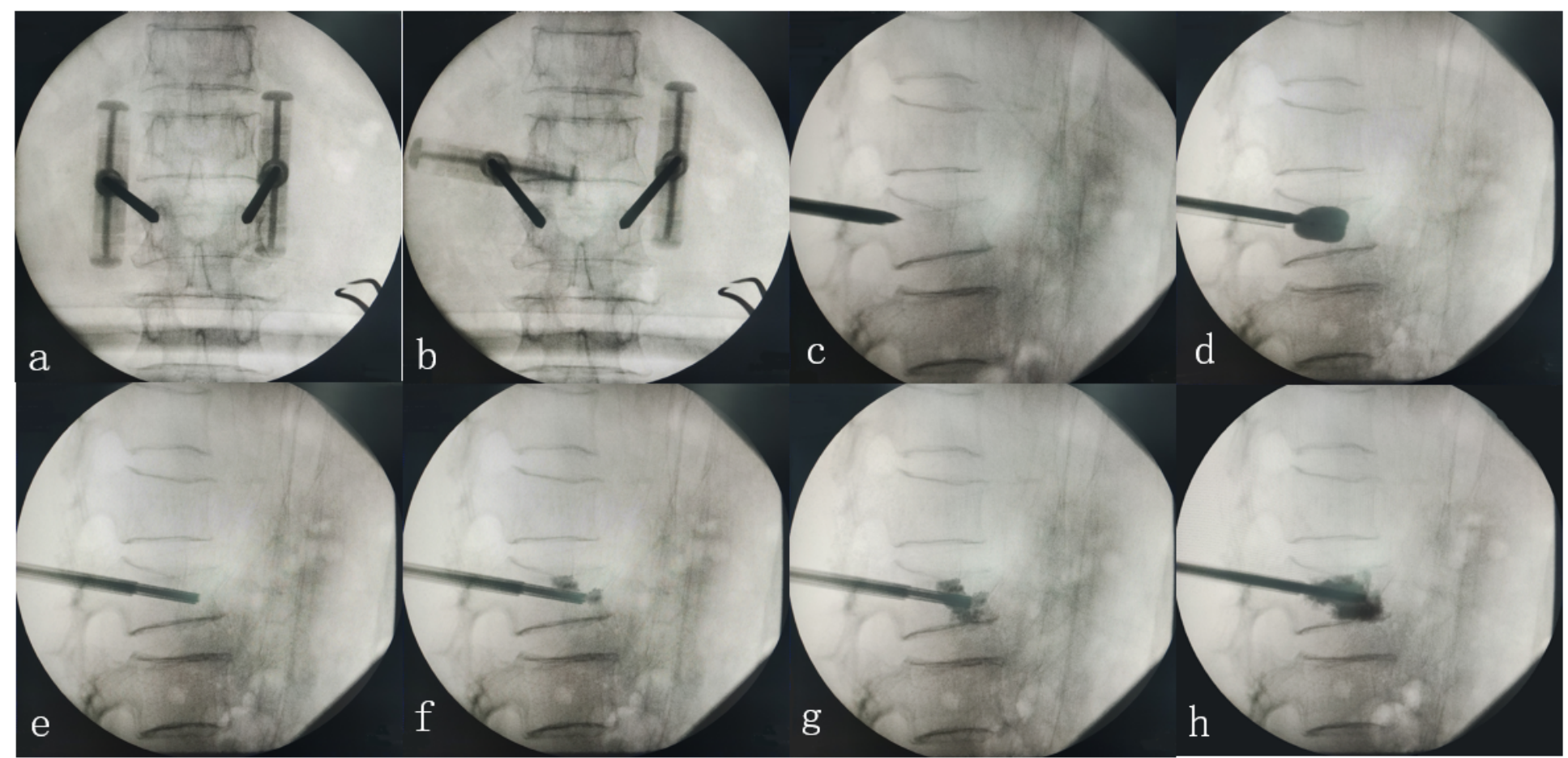

\section{Figure 1}

A 65 year old woman was diagnosed with L2 OVCF. a Puncture. b Needle tip had reached the medial edge of pedicle shadow in the anterior and posterior position. c Needle tip had reached the posterior edge of vertebral body in the lateral position. $d$ Restored the compression fracture and formed a cavity by expandable balloon. e Placed the bone cement filling tube into the collapse part of the bone cortex. $f$ Injected the bone cement when the bone cement was in the "dough stage". g-h Injected the bone cement into all directions of the vertebral body when the bone cement was in the "wire drawing stage". 


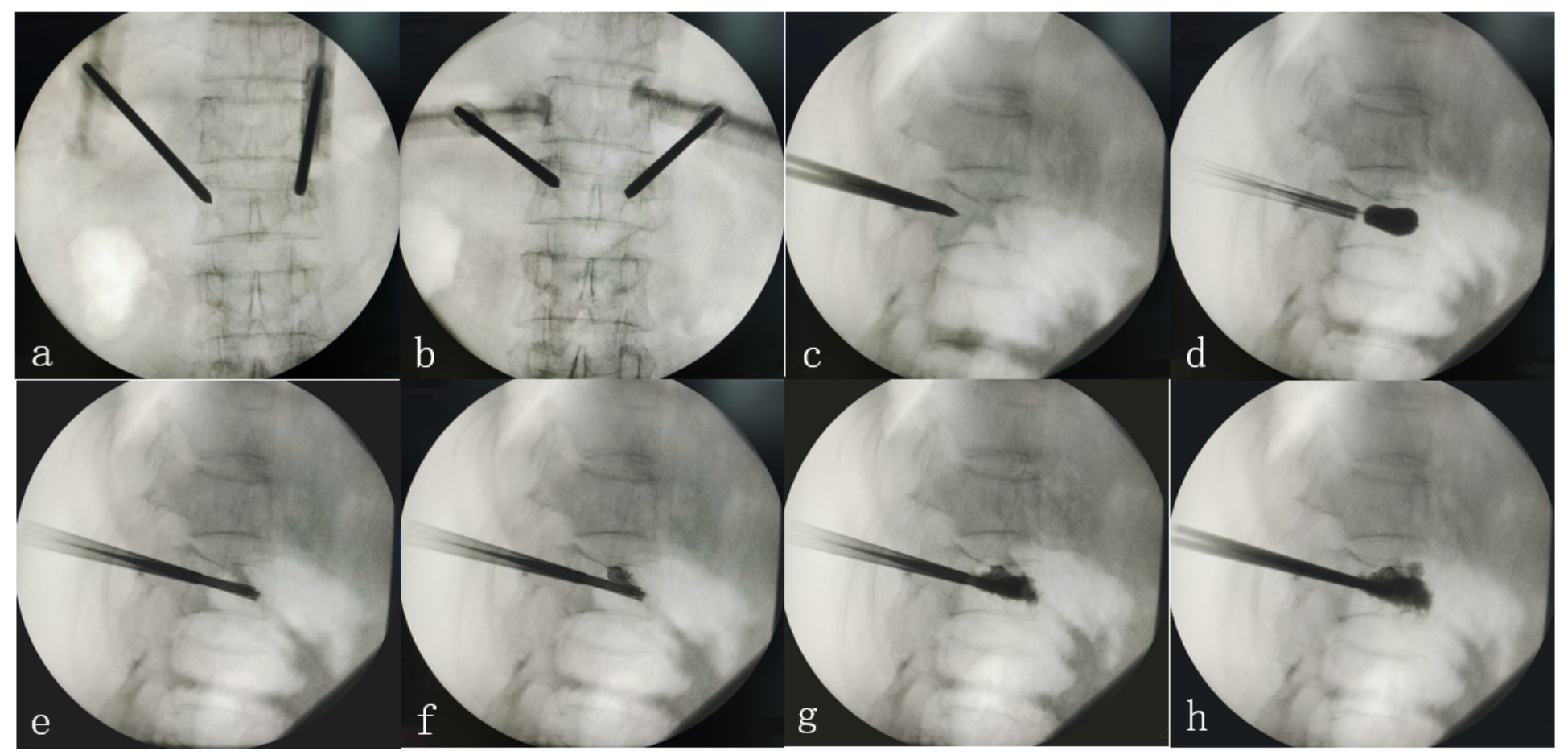

Figure 2

A 71 year old woman was diagnosed with T12 OVCF. a Puncture. b Needle tip had reached the medial edge of pedicle shadow in the anterior and posterior position. $c$ Needle tip had reached the posterior edge of vertebral body in the lateral position. $d$ Restored the compression fracture and formed a cavity by expandable balloon. e Placed the bone cement filling tube into the collapse part of the bone cortex. $f$ Injected the bone cement when the bone cement was in the "dough stage". g-h Injected the bone cement into all directions of the vertebral body when the bone cement was in the "wire drawing stage". 